MATHEMATICS OF COMPUTATION

Volume 78, Number 265, January 2009, Pages 421-429

S 0025-5718(08)02147-9

Article electronically published on September 5, 2008

\title{
SUPERHARMONIC NUMBERS
}

\author{
GRAEME L. COHEN
}

\begin{abstract}
Let $\tau(n)$ denote the number of positive divisors of a natural number $n>1$ and let $\sigma(n)$ denote their sum. Then $n$ is superharmonic if $\sigma(n) \mid n^{k} \tau(n)$ for some positive integer $k$. We deduce numerous properties of superharmonic numbers and show in particular that the set of all superharmonic numbers is the first nontrivial example that has been given of an infinite set that contains all perfect numbers but for which it is difficult to determine whether there is an odd member.
\end{abstract}

\section{INTRODUCTION}

If the harmonic mean of the positive divisors of a natural number $n>1$ is an integer, then $n$ is said to be harmonic. Equivalently, $n$ is harmonic if $\sigma(n) \mid n \tau(n)$, where $\tau(n)$ and $\sigma(n)$ denote the number of positive divisors of $n$ and their sum, respectively. Harmonic numbers were introduced by Ore [8], and named (some 15 years later) by Pomerance 9 .

Harmonic numbers are of interest in the investigation of perfect numbers (numbers $n$ for which $\sigma(n)=2 n$ ), since all perfect numbers are easily shown to be harmonic. All known harmonic numbers are even. If it could be shown that there are no odd harmonic numbers, then this would solve perhaps the most longstanding problem in mathematics: whether or not there exists an odd perfect number. Recent articles in this area include those by Cohen and Deng [3] and Goto and Shibata [5].

In [3], the following was proposed as a scheme to demonstrate the nonexistence of odd perfect numbers:

Let $P_{0}$ be the set of perfect numbers and devise a finite sequence $P_{1}, \ldots, P_{z}$ of sets of natural numbers such that

- $P_{0} \subset P_{1} \subset \cdots \subset P_{z}$, all inclusions being strict,

- it seems to be difficult to find odd members of $P_{1}, \ldots, P_{z-1}$ as each is constructed, in turn,

- it can be proved that there are no odd members of $P_{z}$.

(It would be understood that the sets $P_{1}, P_{2}, \ldots$ must not be trivially or artificially defined, for example by letting $P_{1}$ be the union of the set of all even numbers and the set of all perfect numbers.)

Let $P_{1}$ stand for the set of harmonic numbers. In [3], it was suggested that a candidate for $P_{2}$ could be the set of all numbers $n>1$ such that $\sigma_{k}(n) \mid n^{k} \tau(n)$ where $k$ is a positive integer and $\sigma_{k}(n)$ denotes the sum of the $k$ th powers of the

Received by the editor April 12, 2007 and, in revised form, January 22, 2008.

2000 Mathematics Subject Classification. Primary 11A25, 11 Y70.

(C)2008 American Mathematical Society 
positive divisors of $n$. For such a number, the harmonic mean of the $k$ th powers of the divisors of $n$ is an integer. Although a number of properties of this $P_{2}$ were obtained, the authors could not show that the inclusion $P_{1} \subset P_{2}$ is strict. The article also described other examples from the literature that could be specified as $P_{1}$, such as the set of multiperfect numbers, that is, natural numbers $n>1$ for which $\sigma(n)=l n$, for some positive integer $l$.

We observe at this point that there are 30 harmonic numbers up to $10^{6}$, of which just four are perfect numbers. It is not known, however, whether the set $P_{1}$ of harmonic numbers is infinite or not. In the present paper, a different generalisation of this set $P_{1}$ is given which, in stark contrast to the set $P_{2}$ just described, is readily shown to be infinite. Up to $10^{6}$, it contains 3453 members, all of them even. If it can be shown that this set contains no odd members, then it would follow that there are no odd perfect numbers. More data will be given below.

\section{Superharmonic Numbers}

Our new candidate for $P_{2}$ is the set of superharmonic numbers: natural numbers $n>1$ for which $\sigma(n) \mid n^{k} \tau(n)$, for some positive integer $k$. If $n$ is superharmonic, then the smallest $k$ such that $\sigma(n) \mid n^{k} \tau(n)$ is called the index of $n$, denoted by $\underline{k}(n)$. Harmonic numbers have index 1 . Table 1 gives the number of superharmonic numbers in three intervals of length $2 \cdot 10^{8}$, counted according to their index; all 141,336 superharmonic numbers in these intervals are even. Table 2 gives the smallest superharmonic numbers with index $1,2, \ldots, 24$.

Regarding notation, roman letters always denote integers, with $p$ and $q$ reserved for primes. We write $v_{p}(m)$ for the exponent on $p$ in $m$, that is, the integer $a \geq 0$ such that $p^{a} \mid m$ and $p^{a+1} \nmid m$. It is often convenient to write $p^{a} \| m$ when $v_{p}(m)=a$.

We recall that, if $n=\prod p^{a}$ represents the canonical decomposition of $n$ into primes, then

$$
\tau(n)=\prod(a+1), \quad \sigma(n)=\prod\left(1+p+p^{2}+\cdots+p^{a}\right)=\prod \frac{p^{a+1}-1}{p-1}
$$

The functions $\tau$ and $\sigma$ are multiplicative.

The definition of a superharmonic number does not provide an effective search technique when $n$ is very large, as in later examples (see Table 3 ) where we have $n$ around $10^{1500}$, with $\underline{k}(n)$ exceeding 490 . Our first result is far more useful in the search for numerical information.

Theorem 1. Let $n$ be a superharmonic number. Then, for each prime divisor $p$ of $\sigma(n), p \mid n \tau(n)$. Further, if $v_{p}(\sigma(n))>v_{p}(\tau(n))$, then $p \mid n$. The index of $n$ is given by

$$
\underline{k}(n)=\max _{\substack{p \mid \sigma(n) \\ v_{p}(n) \neq 0}}\left\lceil\frac{v_{p}(\sigma(n))-v_{p}(\tau(n))}{v_{p}(n)}\right\rceil
$$


TABLE 1. The number of superharmonic numbers, counted according to their index $\underline{k}$, in each of the three given intervals.

\begin{tabular}{|rrcc|}
\hline$\underline{k}$ & {$\left[2,2 \cdot 10^{8}\right]$} & {$\left[10^{9}+1,10^{9}+2 \cdot 10^{8}\right]$} & {$\left[10^{10}+1,10^{10}+2 \cdot 10^{8}\right]$} \\
\hline 1 & 81 & 3 & 0 \\
2 & 6317 & 1062 & 292 \\
3 & 14689 & 3708 & 1255 \\
4 & 16998 & 4868 & 1773 \\
5 & 15804 & 4997 & 2032 \\
6 & 13524 & 4315 & 1802 \\
7 & 10379 & 3667 & 1691 \\
8 & 7325 & 3243 & 1297 \\
9 & 4658 & 1940 & 1067 \\
10 & 2857 & 1253 & 692 \\
11 & 1683 & 880 & 508 \\
12 & 994 & 690 & 326 \\
13 & 572 & 268 & 159 \\
14 & 344 & 183 & 130 \\
15 & 172 & 152 & 114 \\
16 & 101 & 105 & 72 \\
17 & 54 & 28 & 29 \\
18 & 42 & 23 & 14 \\
19 & 13 & 21 & 15 \\
20 & 11 & 8 & 10 \\
21 & 3 & 0 & 4 \\
22 & 4 & 3 & 3 \\
23 & 0 & 7 & 2 \\
\hline Totals & 96625 & 31424 & 13287 \\
\hline
\end{tabular}

Proof. The first two statements follow from the definition of a superharmonic number, since an integer $k$ exists such that $p^{a} \mid n^{k} \tau(n)$ for each $p$, where $a=v_{p}(\sigma(n))$. Then also

$$
k v_{p}(n)+v_{p}(\tau(n)) \geq v_{p}(\sigma(n)) .
$$

Let $k^{\prime}(p)$ be the smallest $k$ for which this holds. We set $k^{\prime}(p)=0$ if $v_{p}(n)=0$ and observe that $v_{p}(n)>0$ for at least one $p$, since $\sigma(n)>\tau(n)$. Then $\sigma(n) \mid n^{k} \tau(n)$ provided $k \geq k^{\prime}(p)$ for all $p$. The result follows.

It is easy to see, as Ore 8 showed for harmonic numbers, that a single prime power cannot be superharmonic. The proof by Callan [2] (see also Pomerance [9]) that the only harmonic numbers with two distinct prime factors are the even perfect numbers does not carry through to superharmonic numbers although it would seem to be true.

The next theorem quickly implies that there are infinitely many superharmonic numbers.

Theorem 2. Let $q_{j}$ denote the $j$ th prime $\left(q_{1}=2, q_{2}=3, \ldots\right)$ and put $M_{j}=$ $q_{1} q_{2} \cdots q_{j}$. Then $M_{j}$ is superharmonic for all $j \geq 2$. 
TABLE 2. The smallest superharmonic number $n$ with index $\underline{k}(n)$. If $\underline{k}(n) \geq 25$, then $n>10^{9}$.

\begin{tabular}{|rl||rl|}
\hline$\underline{k}(n)$ & $n$ & $\underline{k}(n)$ & $n$ \\
\hline 1 & $6=2 \cdot 3$ & 13 & $540606=2 \cdot 3 \cdot 11 \cdot 8191$ \\
2 & $30=2 \cdot 3 \cdot 5$ & 14 & $344022=2 \cdot 3 \cdot 7 \cdot 8191$ \\
3 & $102=2 \cdot 3 \cdot 17$ & 15 & $2309862=2 \cdot 3 \cdot 47 \cdot 8191$ \\
4 & $186=2 \cdot 3 \cdot 31$ & 16 & $786426=2 \cdot 3 \cdot 131071$ \\
5 & $1146=2 \cdot 3 \cdot 191$ & 17 & $4718586=2 \cdot 3 \cdot 786431$ \\
6 & $762=2 \cdot 3 \cdot 127$ & 18 & $3145722=2 \cdot 3 \cdot 524287$ \\
7 & $8022=2 \cdot 3 \cdot 7 \cdot 191$ & 19 & $33030102=2 \cdot 3 \cdot 7 \cdot 786431$ \\
8 & $5334=2 \cdot 3 \cdot 7 \cdot 127$ & 20 & $22020054=2 \cdot 3 \cdot 7 \cdot 524287$ \\
9 & $35526=2 \cdot 3 \cdot 31 \cdot 191$ & 21 & $146276166=2 \cdot 3 \cdot 31 \cdot 786431$ \\
10 & $23622=2 \cdot 3 \cdot 31 \cdot 127$ & 22 & $97517382=2 \cdot 3 \cdot 31 \cdot 524287$ \\
11 & $145542=2 \cdot 3 \cdot 127 \cdot 191$ & 23 & $599260422=2 \cdot 3 \cdot 127 \cdot 786431$ \\
12 & $49146=2 \cdot 3 \cdot 8191$ & 24 & $399506694=2 \cdot 3 \cdot 127 \cdot 524287$ \\
\hline
\end{tabular}

Proof. The number 2 is not superharmonic and the number $2 \cdot 3$ is (see Table 2). For $j \geq 3$, consider the quotient

$$
\begin{aligned}
\theta=\frac{M_{j}^{k} \tau\left(M_{j}\right)}{\sigma\left(M_{j}\right)} & =\frac{\left(2 \cdot 3 q_{3} q_{4} \cdots q_{j}\right)^{k} \cdot 2^{j}}{\sigma\left(2 \cdot 3 q_{3} q_{4} \cdots q_{j}\right)} \\
& =\frac{\left(2 \cdot 3 q_{3} q_{4} \cdots q_{j}\right)^{k}}{3 \cdot \frac{3+1}{2^{2}} \frac{q_{3}+1}{2} \frac{q_{4}+1}{2} \cdots \frac{q_{j}+1}{2}}
\end{aligned}
$$

where we have used the multiplicativity of $\tau$ and $\sigma$. Every prime divisor of the denominator is less than $q_{j}$ and hence the denominator divides $M_{j}^{k}$ for suitably large $k$. For such $k, \theta$ is an integer, so $M_{j}$ is superharmonic.

Corollary 3. There are infinitely many superharmonic numbers. Furthermore, the set $\{\underline{k}(n): n$ superharmonic $\}$ of all possible indices is infinite.

Proof. There are infinitely many superharmonic numbers because there are infinitely many prime numbers. Moreover, there are infinitely many primes congruent to $3(\bmod 4)$, so $v_{2}\left(\sigma\left(M_{j}\right)\right)$ increases indefinitely with $j$. Then $k$ must be increasingly large, as $j$ increases, to allow $\theta$ to be an integer. This implies the second statement of the corollary.

To illustrate the growth of $\underline{k}\left(M_{j}\right)$ with $j$, its values are given in Table 3 for $2 \leq j \leq 40$ and $493 \leq j \leq 500$. The sequence $\left\{\underline{k}\left(M_{j}\right)\right\}_{j \geq 2}$ is necessarily increasing, but Table 3 shows that it is not strictly increasing.

The following result shows that every natural number divides a superharmonic number. This provides a second proof that there are infinitely many such numbers.

Theorem 4. For any natural number $N$, there exists a superharmonic number which is a multiple of $N$.

Proof. Set $N_{0}=N$ if $N$ is even and $N_{0}=2 N$ if $N$ is odd. Suppose $N_{0}$ is not superharmonic. Let $p_{1}$ be the smallest prime such that $p_{1} \mid \sigma\left(N_{0}\right)$ and $p_{1} \nmid N_{0}$; put $N_{1}=N_{0} p_{1}$. Let $p_{2}$ be the smallest prime such that $p_{2} \mid \sigma\left(N_{1}\right)$ and $p_{2} \nmid N_{1}$; put 
TABLE 3 . The index $\underline{k}\left(M_{j}\right)$ of $M_{j}$, the product of the first $j$ primes, for $2 \leq j \leq 40$ and $493 \leq j \leq 500$.

\begin{tabular}{|rcc||rcc||rcc|}
\hline$j$ & $\underline{k}\left(M_{j}\right)$ & $\log _{10}\left(M_{j}\right)$ & $j$ & $\underline{k}\left(M_{j}\right)$ & $\log _{10}\left(M_{j}\right)$ & $j$ & $\underline{k}\left(M_{j}\right)$ & $\log _{10}\left(M_{j}\right)$ \\
\hline 2 & 1 & 0.778 & 18 & 15 & 23.069 & 34 & 33 & 55.001 \\
3 & 2 & 1.477 & 19 & 16 & 24.895 & 35 & 33 & 57.174 \\
4 & 2 & 2.322 & 20 & 18 & 26.747 & 36 & 35 & 59.353 \\
5 & 3 & 3.364 & 21 & 18 & 28.610 & 37 & 35 & 61.549 \\
6 & 3 & 4.478 & 22 & 21 & 30.508 & 38 & 36 & 63.761 \\
7 & 5 & 5.708 & 23 & 22 & 32.427 & 39 & 38 & 65.984 \\
8 & 5 & 6.987 & 24 & 22 & 34.376 & 40 & 38 & 68.222 \\
9 & 6 & 8.348 & 25 & 22 & 36.363 & $\ldots$ & & \\
10 & 7 & 9.811 & 26 & 22 & 38.367 & 493 & 486 & 1494.895 \\
11 & 10 & 11.302 & 27 & 24 & 40.380 & 494 & 486 & 1498.443 \\
12 & 10 & 12.870 & 28 & 25 & 42.409 & 495 & 487 & 1501.992 \\
13 & 10 & 14.483 & 29 & 25 & 44.447 & 496 & 487 & 1505.541 \\
14 & 11 & 16.117 & 30 & 25 & 46.500 & 497 & 488 & 1509.091 \\
15 & 14 & 17.789 & 31 & 31 & 48.604 & 498 & 488 & 1512.642 \\
16 & 14 & 19.513 & 32 & 32 & 50.721 & 499 & 490 & 1516.194 \\
17 & 15 & 21.284 & 33 & 32 & 52.858 & 500 & 491 & 1519.746 \\
\hline
\end{tabular}

$N_{2}=N_{1} p_{2}$. Let $p_{3}$ be the smallest prime such that $p_{3} \mid \sigma\left(N_{2}\right)$ and $p_{3} \nmid N_{2}$, and continue in this fashion until the process ends with a prime $p_{j}$ such that $p_{j} \mid \sigma\left(N_{j-1}\right)$ and $p_{j} \nmid N_{j-1}$. This end will occur when all prime divisors of $\sigma\left(p_{j}\right)=p_{j}+1$, being less than $p_{j}$, occur among $p_{1}, \ldots, p_{j-1}$ and the prime divisors of $N_{0}$. Note that $2 \nmid p_{1} p_{2} \cdots p_{j}$. Put $N_{j}=N_{j-1} p_{j}$ and consider the quotient

$$
\begin{aligned}
\theta=\frac{N_{j}^{k} \tau\left(N_{j}\right)}{\sigma\left(N_{j}\right)} & =\frac{\left(N_{0} p_{1} p_{2} \cdots p_{j}\right)^{k} \cdot 2^{j} \tau\left(N_{0}\right)}{\sigma\left(N_{0} p_{1} p_{2} \cdots p_{j}\right)} \\
& =\frac{\left(N_{0} p_{1} p_{2} \cdots p_{j}\right)^{k} \tau\left(N_{0}\right)}{\sigma\left(N_{0}\right) \frac{p_{1}+1}{2} \frac{p_{2}+1}{2} \cdots \frac{p_{j}+1}{2}} .
\end{aligned}
$$

We have again used the multiplicativity of $\tau$ and $\sigma$. By construction, every prime factor of the denominator divides $N_{0} p_{1} p_{2} \cdots p_{j}$ so that, for suitably large $k$, the numerator of $\theta$ is divisible by its denominator. For such $k, \theta$ is an integer, so $N_{j}$ is superharmonic.

\section{OdD SUPERHARMONIC NUMBERS}

There are infinitely many superharmonic numbers, and all of those known to date are even. Furthermore, there are infinitely many superharmonic numbers that are squarefree, that is, not divisible by $p^{2}$ for any prime $p$, as is clear from Theorem 2 . But, as we show below, no odd superharmonic number, if there is one, can be squarefree. Ore [8] proved the corresponding result for harmonic numbers long ago. Another long-established result for harmonic numbers, due to Garcia [4], is that if $n$ is odd and harmonic and $p^{a} \| n$, then $p^{a} \equiv 1(\bmod 4)$. These properties for superharmonic numbers are given in the following theorem. 
Theorem 5. Let $N$ be an odd superharmonic number. Then (i) $N$ cannot be squarefree, (ii) if $p^{a} \| n$, then $p^{a} \equiv 1(\bmod 4)$.

Proof. (i) Suppose $N=p_{1} p_{2} \cdots p_{j}$ for odd primes $p_{1}<p_{2}<\cdots<p_{j}$. Consider the quotient

$$
\theta=\frac{N^{k} \tau(N)}{\sigma(N)}=\frac{\left(p_{1} \cdots p_{j}\right)^{k} \cdot 2^{j}}{\sigma\left(p_{1} \cdots p_{j}\right)}=\frac{\left(p_{1} \cdots p_{j}\right)^{k}}{\frac{p_{1}+1}{2} \cdots \frac{p_{j}+1}{2}} .
$$

The prime factors of $\left(p_{1}+1\right) / 2$ are less than $p_{1}$, so the denominator cannot be a factor of the numerator, for any $k$. Then $\theta$ cannot be an integer, so $N$ is not superharmonic.

(ii) Suppose $p^{a} \not \equiv 1(\bmod 4)$, so that $p \equiv 3(\bmod 4)$ and $a$ is odd. Write $a=2^{j} b-1$, where $b \geq 1$ is odd and $j \geq 1$, and consider the factorisation

$$
\begin{aligned}
\sigma\left(p^{a}\right) & =\frac{p^{a+1}-1}{p-1}=\frac{\left(p^{b}\right)^{2^{j}}-1}{p-1} \\
& =\frac{p^{b}-1}{p-1}\left(p^{b}+1\right)\left(p^{2 b}+1\right)\left(p^{2^{2} b}+1\right) \cdots\left(p^{2^{j-1} b}+1\right) .
\end{aligned}
$$

Since $p^{b} \equiv 3(\bmod 4)$, this implies that $v_{2}\left(\sigma\left(p^{a}\right)\right) \geq j+1$, whereas $v_{2}\left(\tau\left(p^{a}\right)\right)=j$. On the other hand, if $p^{a} \equiv 1(\bmod 4)$, then $v_{2}\left(\sigma\left(p^{a}\right)\right)=v_{2}\left(\tau\left(p^{a}\right)\right)$. So $v_{2}(\sigma(N))>$ $v_{2}(\tau(N))$ if $p^{a} \equiv 3(\bmod 4)$ for at least one prime factor $p$ of $N$. In that case, $n^{k} \tau(N) / \sigma(N)$ cannot be an integer, for any $k$, so $N$ is not superharmonic. The result follows.

Ore's proof of the harmonic version of (i) was different from the above. By his approach, he was able to show further that the only squarefree harmonic number, odd or even, is 6 . Of course, that does not extend to superharmonic numbers. The proof given here of (ii) is essentially Garcia's.

As one consequence of Theorem 5 , not previously noted for harmonic numbers, we have the following. The proof is omitted.

Corollary 6. Suppose $p^{2} M$ is an odd superharmonic number, where $M$ is squarefree and not divisible by the prime $p$. Then $p \equiv \pm 1(\bmod 12)$.

\section{Conclusion. Thoughts on odd Perfect numbers}

To return to the scheme outlined in the Introduction, let $P_{1}$ be the set of harmonic numbers and $P_{2}$ the set of superharmonic numbers. The earlier attempt, in [3, to find a nontrivial set that extends $P_{1}$, yet for which there seem to be no odd members, was not satisfactory for the more general purpose, nor are such extensions as the set of natural numbers $n>1$ for which $\sigma(n) \mid n \tau^{2}(n)$, since 3 is a member of this set. Superharmonic numbers were arrived at by wondering first about numbers $n>1$ for which $\sigma(n) \mid n^{2} \tau(n)$.

The set $P_{0}$ of perfect numbers may well be infinite. Realistically, this depends on showing that the set of Mersenne primes (primes of the form $2^{p}-1$ ) is infinite. It is unlikely that any other approach could be used to show that $P_{1}$ is infinite, also. So the fact that $P_{2}$ is provably infinite is a bonus.

What about the density of $P_{2}$ ? The density of a set $P$ is

$$
\delta_{P}=\lim _{x \rightarrow \infty} \frac{N_{P}(x)}{x},
$$


if the limit exists, where $x$ is a real variable and $N_{P}(x)$ is the number of elements of $P$ not exceeding $x$. It was shown by Kanold [6] that $P_{1}$ has zero density, but a similar argument does not carry through to $P_{2}$ (nor to the set of superharmonic numbers of index $k$, for any given $k>1$ ), but see the Appendix. The preferred next step in this scheme is to seek $P_{3}$, such that $P_{2} \subset P_{3}, P_{3}$ has no apparent odd members, and $\delta_{P_{2}}<\delta_{P_{3}}$.

Compared to superharmonic numbers, the tighter definition of odd perfect numbers allows more to be determined regarding their prime factor decomposition, if there is such a number. In place of Theorem 5 , it has long been known that an odd perfect number must have the form $p_{0}^{b_{0}} p_{1}^{2 b_{1}} p_{2}^{2 b_{2}} \cdots p_{t}^{2 b_{t}}$, where $p_{0} \equiv b_{0} \equiv 1(\bmod 4)$. Furthermore, Nielsen 7 has recently proved that $t \geq 8$, the first improvement on that bound for more than 25 years, and since 1991 it has been known that there are no odd perfect numbers below $10^{300}$ (Brent et al. 1]). Anything at all close to such results for odd superharmonic numbers, or odd harmonic numbers, would be very difficult.

Searches for odd perfect numbers, and more generally for a multiperfect number $n$ (even or odd), must make use of the fact that the set of prime factors of $\sigma(n)$ must almost coincide with the set $Q$ of prime factors of $n$ itself; in particular, if $p^{a} \| n$, then the prime factors of $\sigma\left(p^{a}\right)$ mostly must remain within $Q$. The proofs of most of the theorems in this paper depend essentially on the same requirement. That is, the likelihood of finding an odd superharmonic number would seem to be about the same as that of finding an odd perfect number.

\section{Appendix}

I am grateful to the referee of this paper for the following theorem.

Theorem 7. The set $P_{2}$ of superharmonic numbers has density zero.

Proof. We must show that $\delta_{P_{2}}=0$ or, equivalently, $N_{P_{2}}(x)=o(x)$ as $x \rightarrow \infty$. For any set $P$, write $\#\{n \leq x: n \in P\}$ for $N_{P}(x)$, with variations on this notation that will be obvious. Let $P^{+}(n)$ denote the largest prime factor of an integer $n \geq 2$, and put $P^{+}(1)=1$.

Define

$$
\Psi(x, y)=\#\left\{n \leq x: P^{+}(n) \leq y\right\}, \quad x \geq y \geq 2 .
$$

It is shown in Tenenbaum [10] that, for large $x$,

$$
\Psi(x, y) \ll x e^{-u / 2},
$$

where $u=(\log x) /(\log y)$, uniformly in $y$. Setting $y=\exp \left((\log x)^{2 / 3}\right)$, it follows that $\Psi(x, y)=o(x)$, so to prove that $\delta_{P_{2}}=0$ it suffices to show that

$$
\#\left\{n \leq x: n \in P_{2}, P^{+}(n)>y\right\}=o(x) .
$$

Moreover, since

$$
\#\left\{n \leq x: q=P^{+}(n)>y, q^{2} \mid n\right\} \leq \sum_{y<q \leq x} \sum_{\substack{n \leq x \\ q^{2} \mid n}} 1 \ll \sum_{q>y} \frac{x}{q^{2}} \ll \frac{x}{y},
$$

it suffices to show that

$$
\#\left\{n \leq x: n \in P_{2}, q=P^{+}(n)>y, q \| n\right\}=o(x) .
$$


In fact, it it is enough to show that

$$
\#\left\{n \leq x: n \in P_{2}, q=P^{+}(n)>y, q \| n, P^{+}(q+1)>z\right\}=o(x),
$$

where $z=\exp \left((\log x)^{1 / 3}\right)$. To see why, observe that

$$
\begin{aligned}
\#\left\{n \leq x: q=P^{+}(n)>y, q \| n, P^{+}(q+1) \leq z\right\} & \leq \sum_{\substack{y<q \leq x \\
P^{+}(q+1) \leq z}} \sum_{\substack{n \leq x \\
q \| n}} 1 \\
& \leq x \sum_{\substack{y<q \leq x \\
P^{+}(q+1) \leq z}} \frac{1}{q} \ll x \sum_{\substack{y<n \leq x \\
P^{+}(n) \leq z}} \frac{1}{n} .
\end{aligned}
$$

By partial summation, the last sum is

$$
\int_{y}^{x} \frac{d \Psi(u, z)}{u}=\frac{\Psi(x, z)}{x}-\frac{\Psi(y, z)}{y}+\int_{y}^{x} \frac{\Psi(u, z)}{u^{2}} d u,
$$

and, using the bound in (1), each term on the right is easily seen to be $o(1)$ as $x \rightarrow \infty$. Thus, we need only prove (2).

Let $n$ lie in the subset of $P_{2}$ indicated in (2). Write $n=q m$, where $q=P^{+}(n)$, so $q \nmid m$, and put $r=P^{+}(q+1)$. Then, we have $\sigma(n) \mid n^{k} \tau(n)$ for some $k$ if and only if

$$
(q+1) \sigma(m) \mid 2(q m)^{k} \tau(m) .
$$

Since $q$ and $q+1$ are coprime, it follows that $r \mid 2 m^{k} \tau(m)$. Suppose $r \mid \tau(m)$. Then $p^{r-1} \mid n$ for some prime $p$, so $x \geq n>2^{r-1}>2^{z-1}$, but this is not possible for $z=\exp \left((\log x)^{1 / 3}\right)$. Therefore, $r \nmid \tau(m)$, and $r \neq 2$, so $r \mid m$. That is, $q P^{+}(q+1) \mid n$. But the number of such integers $n$ up to $x$ does not exceed

$$
\sum_{\substack{y<q \leq x \\ P^{+}(q+1)>z}} \frac{x}{q P^{+}(q+1)}<\frac{x}{z} \sum_{\substack{y<q \leq x \\ P^{+}(q+1)>z}} \frac{1}{q}<\frac{x}{z} \sum_{n \leq x} \frac{1}{n} \ll \frac{x \log x}{z}=o(x),
$$

which proves (2).

\section{REFERENCES}

[1] R. P. Brent, G. L. Cohen and H. J. J. te Riele, "Improved techniques for lower bounds for odd perfect numbers", Math. Comp., 57 (1991), 857-868. MR1094940 (92c:11004)

[2] D. Callan, Problems and Solutions: Solutions: 6616, Amer. Math. Monthly, 99 (1992), 783789. MR,1542194

[3] G. L. Cohen and Moujie Deng, "On a generalisation of Ore's harmonic numbers", Nieuw Archief voor Wiskunde, 16 (1998), 161-172. MR.1680101(2000k:11008)

[4] M. Garcia, "On numbers with integral harmonic mean", Amer. Math. Monthly, 61 (1954), 89-96. MR0059291 (15:506d)

[5] T. Goto and S. Shibata, "All numbers whose positive divisors have integral harmonic mean up to 300", Math. Comp., 73 (2004), 475-491. MR2034133 (2004j:11005)

[6] H.-J. Kanold, "Über das harmonische Mittel der Teiler einer natürlichen Zahl", Math. Ann., 133 (1957), 371-374. MR0089219 (19:635f)

[7] P. P. Nielsen, "Odd perfect numbers have at least nine distinct prime factors", Math. Comp., 76 (2007), 2109-2126. MR22336286

[8] O. Ore, "On the averages of the divisors of a number", Amer. Math. Monthly, 55 (1948), 614-619. MR0027292(10:284a) 
[9] C. Pomerance, On a problem of Ore: Harmonic numbers, unpublished manuscript (1973).

[10] G. Tenenbaum, Introduction to Analytic and Probabilistic Number Theory, Cambridge University Press, Cambridge (1995). MR1342300(97e:11005b)

Department of Mathematical Sciences, University of Technology, Sydney, BroadWAY, NSW 2007, Australia

Current address: 1201/95 Brompton Road, Kensington, NSW 2033, Australia

E-mail address: g.cohen@bigpond.net.au 